\title{
Efficiency of Chemical Ripener Action in Sugareane. IV. Growth and Qualitative Responses to Polaris Under Variable Solar Radiation Regimes ${ }^{1}$
}

\begin{abstract}
Alex G. Alexander ${ }^{2}$
ABSTRACT

Growth and qualitative effects of Polaris (N,N-bis [phosphono methyl]glycine) were evaluated in early-adult sugarcane receiving variable levels of solar radiation. Three experiments were performed in which plants initially propagated under clear glass received light, intermediate, and heavy shade regimes offering maximum light intensities of 5600,2300 , and $450 \mathrm{fc}$, respectively. Ripener treatments were given simultaneously in ful! sunlight (daily $\max .=9600 \mathrm{fc}$ ). High sunlight intensities were not required for optimal Polaris action, and Polaris did not improve the plants' utilization of low light intensities. Growth repression under light- and intermediate-shade regimes was comparable to that attained under full sunlight. Maximum juice quality was produced under intermediate shade, while heavy shade eliminated entirely the ripener's qualitative effects. Results suggest that Polaris requires light at relatively low intensities for ripening processes not directly dependent upon photosynthesis, and that it can be used effectively under natural cloud cover restricting solar radiation levels to 2000-3000 fc.
\end{abstract}

\section{INTRODUCTION}

With the advent of Polaris ${ }^{3,4}$ as the first commercial chemical ripener for sugarcane, a series of ripener efficiency studies was begun in Puerto Rico using Polaris as a model compound. The series is intended, first, to identify the ripener's physiological and administrative requirements for maximum effectiveness, and, second, to establish a research-procedural model by which the requirements of succeeding or alternate ripeners are methodically determined.

Recent findings suggest that the long term effectiveness of Polaris is determined early in its treatment interval (4), and that the period of leaf illumination immediately preceding the chemical's reception may be decisive (3). On the other hand, little is known of the light intensity requirements for optimal action once the ripener is incorporated into the sugarcane source-to-sink system. Whether or not Polaris can enhance

1 Manuseript submitted to the Editorial Board January 29, 1976.

2 Plant Physiologist, Agricultural Experiment Station, University of Puerto Rico, Mayagüez Campus, Río Piedras, P.R.

${ }^{3}$ N,N-bis(phosphonomethyl)glycine. Monsanto Agricultural Products Co., St. Louis, Mo.

4 Trade names are used in this publication solely for the purpose of providing specific information. Mention of a trade name does not constitute a guarantee or warranty of equipment or materials by the Agricultural Experiment Station of the University of Puerto Rico or an endorsement over other equipment or materials not mentioned. 
the utilization of the abundant sunlight normally received during Puerto Rico's dry season or perform with full effectiveness during periods of extended cloud cover are questions remaining to be answered. The performance of Polaris within discrete regimes of solar radiation is presented in this report.

\section{MATERIALS AND METHODS}

Three variable-sunlight experiments were performed with 12- to 14week-old plants of the interspecific hybrid PR 980. All were propagated by sand culture as previously described (1). The two initial experiments
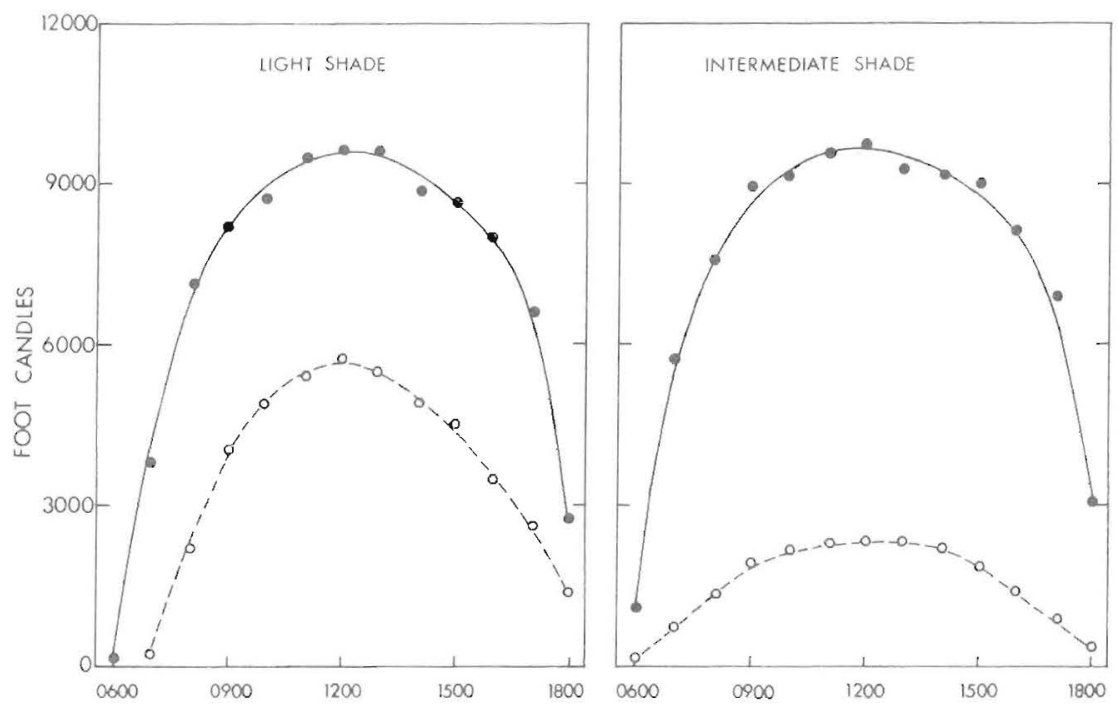

HOUR

Fig. 1.-Diurnal solar radiation values received under full sunlight (through unpainted glass) and under the light- and intermediate-shade regimes produced for experiments 1 and 2. Symbols: (--) full sunlight; (--O--) shade.

used an adjacent pair of identically-designed greenhouses, one of which was coated with successive single and double layers of white, allweather latex paint. The second greenhouse served as the sunlight control, or "full sun" facility, for all experiments. Figure 1 illustrates diumal radiation curves for experiments 1 and 2. Maximum radiation. values for the light- and intermediate-shade regimes were in the order of 5600 and $2300 \mathrm{fc}$, respectively. For the third experiment, a sunlightdeficiency regime ("heavy shade") was produced with a fully ventilated warehouse illuminated through side and overhead windows. Maximum light intensity was approximately $450 \mathrm{fc}$. These facilities were situated in a natural air-drainage channel where ventilation was excellent on 
virtually a $24 \mathrm{~h}$ basis. As a consequence, temperature differentials between full sun and shaded areas were small and not taken to be a compromising factor.

Successive plant populations for the three experiments were propagated under clear glass. At 12 days prior to ripener application, half of the plants were transferred to the respective shaded structure for equilibration within the new light regime. Ripener treatments were established in both full sun and shade facilities at $0700 \mathrm{~h}$ on day "0". These consisted of aqueous Polaris solutions containing $3000 \mathrm{p} / \mathrm{m}$ active ingredient, plus distilled-water controls, each containing $0.10 \%$ Tween 20 as wetting agent. There were three replications of each treatment arranged in an incomplete randomized block design.

Samples consisting of six uniform plants/replicate were harvested at $0630 \mathrm{~h}$ on day " 0 ", and at the same hour 21 days thereafter. Green weight was measured for whole plants, tops, and millable stems. Stalks were frozen for subsequent milling and juice quality analyses. At 21 days, leaf number and area measurements were taken for experiments 2 and 3, where some evidence of foliar injury was visible. Chlorophyll content was determined for leaf blade +1 in accordance with Arnon (5). Data were statistically analyzed by the Duncan New Multiple Range test.

\section{RESULTS}

Growth and juice quality values indicate that high light intensity is not a decisive factor for Polaris in its action as a growth repressant and ripener (tables 1 and 2). The magnitude of green-weight repression within light- and intermediate-shade regimes was roughly comparable to the changes produced in full sunlight (table 1). Juice quality improvement was moderately reduced under light shade but increased under intermediate shade (table 2).

The sunlight-deficiency regime of experiment 3 reduced the growth repression and totally eliminated the quality improvement action of Polaris (tables 1 and 2). Polaris treated plants were therefore unable to make more efficient use of a limited light supply.

\section{DISCUSSION}

\section{RIPENING PERSISTENCE UNDER RESTRICTED SOLAR RADIATION}

The light-shade and heavy-shade regimes used in experiments 1 and 3 are relatively extreme conditions, actually experienced by the plant only briefly during morning and evening changes in its sunlight reception curves. On the other hand, the intermediate-shade regime of experiment 2 is a close simulation of field reality, where cloud cover easily reduces light intensities from an optimal 9,000-10,000 fc to a gloomy and shadowless 2000-3000 fc. A case in point was the prolonged rains and 
TABLE 1.-Growth responses of early-adult sugarcane treated with Polaris under variable sunlight regimes

\begin{tabular}{|c|c|c|c|c|c|c|c|c|c|c|c|}
\hline \multirow{3}{*}{$\begin{array}{c}\text { Exp. } \\
\text { no. }\end{array}$} & \multirow{3}{*}{ Light regime } & \multirow{3}{*}{ Treatment ${ }^{1}$} & \multicolumn{9}{|c|}{ Green wt changes ( $\mathrm{g} / \mathrm{plant}$ ) from 0 to 21 days, for- } \\
\hline & & & \multicolumn{3}{|c|}{ Total green weight } & \multicolumn{3}{|c|}{ Top weight } & \multicolumn{3}{|c|}{ Stem weight } \\
\hline & & & 0 & 21 & $\%$ change & 0 & 21 & $\%$ change & 0 & 21 & \% change \\
\hline \multirow[t]{4}{*}{1} & Full sun & Control & $193 \mathrm{a}^{2}$ & $247 \mathrm{a}$ & $28.2 \mathrm{a}$ & $131 \mathrm{a}$ & $158 \mathrm{a}$ & $20.2 \mathrm{a}$ & $62 \mathrm{a}$ & 89 a & $37.8 \mathrm{a}$ \\
\hline & & Polaris & $194 \mathrm{a}$ & $189 \mathrm{~b}$ & $-2.6 \mathrm{~b}$ & $128 \mathrm{a}$ & $104 \mathrm{~b}$ & $-19.1 \mathrm{~b}$ & $66 \mathrm{a}$ & 85 a & $19.4 \mathrm{~b}$ \\
\hline & Light shade & Control & 206 a & 268 a & $30.1 \mathrm{a}$ & $143 \mathrm{a}$ & $178 \mathrm{a}$ & $24.2 \mathrm{a}$ & $63 a$ & $90 \mathrm{a}$ & $43.5 \mathrm{a}$ \\
\hline & & Polaris & $199 \mathrm{a}$ & $183 \mathrm{~b}$ & $-8.0 \mathrm{~b}$ & $136 \mathrm{a}$ & $115 \mathrm{~b}$ & $-15.7 \mathrm{~b}$ & $63 \mathrm{a}$ & $68 \mathrm{~b}$ & $8.6 \mathrm{c}$ \\
\hline \multirow[t]{4}{*}{2} & Full sun & Control & $211 \mathrm{a}$ & 309 a & $46.5 \mathrm{a}$ & $121 \mathrm{a}$ & $168 \mathrm{a}$ & $38.8 \mathrm{a}$ & $90 \mathrm{a}$ & $141 \mathrm{a}$ & $57.6 \mathrm{a}$ \\
\hline & & Polaris & $220 \mathrm{a}$ & $243 \mathrm{~b}$ & $10.5 \mathrm{~b}$ & $124 \mathrm{a}$ & $131 \mathrm{~b}$ & $5.7 \mathrm{~b}$ & $96 \mathrm{a}$ & $112 \mathrm{~b}$ & $16.7 \mathrm{~b}$ \\
\hline & Intermediate & Control & $212 \mathrm{a}$ & $219 \mathrm{~b}$ & $3.3 \mathrm{bc}$ & $121 \mathrm{a}$ & $123 \mathrm{~b}$ & $1.7 \mathrm{~b}$ & $91 \mathrm{a}$ & $96 \mathrm{bc}$ & $4.4 \mathrm{~b}$ \\
\hline & shade & Polaris & $215 \mathrm{a}$ & $176 \mathrm{c}$ & $-18.1 \mathrm{c}$ & $121 \mathrm{a}$ & $91 \mathrm{c}$ & $-24.8 \mathrm{c}$ & $94 \mathrm{a}$ & $85 c$ & $-9.6 \mathrm{~b}$ \\
\hline \multirow[t]{4}{*}{3} & Full sun & Control & $199 \mathrm{a}$ & $283 \mathrm{a}$ & $42.2 \mathrm{a}$ & $135 \mathrm{a}$ & $185 \mathrm{a}$ & $37.0 \mathrm{a}$ & $64 \mathrm{a}$ & 99 a & $54.7 \mathrm{a}$ \\
\hline & & Polaris & $189 \mathrm{a}$ & $205 \mathrm{~b}$ & $8.5 \mathrm{~b}$ & 129 a & $132 \mathrm{~b}$ & $2.3 \mathrm{~b}$ & $59 \mathrm{a}$ & $73 \mathrm{~b}$ & $23.7 \mathrm{~b}$ \\
\hline & Heavy shade & Control & $171 \mathrm{a}$ & $145 \mathrm{c}$ & $-15.2 \mathrm{c}$ & $114 \mathrm{a}$ & $100 \mathrm{bc}$ & $-12.3 \mathrm{c}$ & $58 \mathrm{a}$ & $45 \mathrm{c}$ & $-22.4 \mathrm{c}$ \\
\hline & & Polaris & $177 \mathrm{a}$ & $124 \mathrm{c}$ & $-29.9 \mathrm{~d}$ & $119 \mathrm{a}$ & $82 \mathrm{c}$ & $-31.1 \mathrm{~d}$ & $58 \mathrm{a}$ & $42 \mathrm{c}$ & $-27.6 \mathrm{c}$ \\
\hline
\end{tabular}

${ }^{1}$ Polaris was administered at $0700 \mathrm{~h}$, on day "0", as an aqueous foliar spray containing $3000 \mathrm{p} / \mathrm{m}$ active ingredient.

${ }^{2}$ Values in the same column (within experiments) bearing unlike letters differ significantly $(\mathrm{P}<.05)$. 
cloud cover prevailing in Puerto Rico during November and December of 1975. Within this context the superior ripening activity maintained by Polaris in experiment 2 bears important implications for field usage. Plans to administer Polaris probably should not be curtailed by cloudy weather, and the ripener will likely perform as well during inclement periods as under clear skies.

\section{EVIDENCE OF LIGHT REQUIREMENTS FOR POLARIS ACTION}

The present experiments were established with the view that Polaris may be proportionately more active under higher sunlight intensities. Actually, Polaris responses in full sunlight scarcely equalled those

TABLE 2.-Juice quality responses for early-adult surgarcane treated with Polaris under variable sunlight regimes

\begin{tabular}{|c|c|c|c|c|c|c|c|c|}
\hline \multirow{3}{*}{$\begin{array}{l}\text { Exp. } \\
\text { no. }\end{array}$} & \multirow{3}{*}{ Light regime } & \multirow{3}{*}{$\begin{array}{l}\text { Treat } \\
\text { ment }^{1}\end{array}$} & \multicolumn{6}{|c|}{ Mean values, at 0 and 21 days, for- } \\
\hline & & & \multicolumn{3}{|c|}{ Brix } & \multicolumn{3}{|c|}{ Polarization } \\
\hline & & & 0 & 21 & $\%$ change & 0 & 21 & \% change \\
\hline \multirow[t]{4}{*}{1} & Full sun & Control & $8.5 \mathrm{a}^{2}$ & $9.5 \mathrm{c}$ & $11.8 \mathrm{c}$ & $18.1 \mathrm{a}$ & $23.7 \mathrm{c}$ & $30.9 \mathrm{c}$ \\
\hline & & Polaris & $9.2 \mathrm{a}$ & $15.6 \mathrm{a}$ & $69.6 \mathrm{a}$ & $18.7 \mathrm{a}$ & $48.6 \mathrm{a}$ & $159.9 \mathrm{a}$ \\
\hline & Light shade & Control & $8.4 \mathrm{a}$ & $9.8 \mathrm{c}$ & $16.7 \mathrm{c}$ & $16.3 \mathrm{a}$ & $19.5 \mathrm{c}$ & $19.6 \mathrm{~d}$ \\
\hline & & Polaris & $9.3 \mathrm{a}$ & $13.7 \mathrm{~b}$ & $47.3 \mathrm{~b}$ & $17.6 \mathrm{a}$ & $30.7 \mathrm{~b}$ & $74.4 \mathrm{~b}$ \\
\hline \multirow[t]{4}{*}{2} & Full sun & Control & $10.9 \mathrm{a}$ & $11.9 \mathrm{c}$ & $9.2 \mathrm{~b}$ & $29.0 \mathrm{a}$ & $32.9 \mathrm{~b}$ & $13.5 \mathrm{~b}$ \\
\hline & & Polaris & $10.8 \mathrm{a}$ & $13.6 \mathrm{~b}$ & $25.9 \mathrm{a}$ & $29.5 \mathrm{a}$ & $39.5 \mathrm{ab}$ & $33.9 \mathrm{a}$ \\
\hline & Intermediate & Control & $10.4 \mathrm{a}$ & $11.1 \mathrm{~d}$ & $6.7 \mathrm{~b}$ & $25.2 \mathrm{a}$ & $31.7 \mathrm{~b}$ & $25.8 \mathrm{~b}$ \\
\hline & shade & Polaris & $10.4 \mathrm{a}$ & $14.5 \mathrm{a}$ & $39.4 \mathrm{a}$ & $28.7 \mathrm{a}$ & $46.3 \mathrm{a}$ & $56.8 \mathrm{a}$ \\
\hline \multirow[t]{4}{*}{3} & Full sun & Control & $6.5 \mathrm{a}$ & $7.4 \mathrm{~b}$ & $13.9 \mathrm{~b}$ & $1.3 \mathrm{a}$ & $13.0 \mathrm{~b}$ & $16.5 \mathrm{~b}$ \\
\hline & & Polaris & $7.3 \mathrm{a}$ & $13.2 \mathrm{a}$ & $80.8 \mathrm{a}$ & $12.5 \mathrm{a}$ & $38.9 \mathrm{a}$ & $211.2 \mathrm{a}$ \\
\hline & Heavy shade & Control & $4.7 \mathrm{a}$ & $6.6 \mathrm{~b}$ & $40.3 \mathrm{a}$ & $4.5 \mathrm{~b}$ & $12.9 \mathrm{~b}$ & $186.7 \mathrm{a}$ \\
\hline & & Polaris & $3.9 \mathrm{a}$ & $4.1 \mathrm{~b}$ & $5.1 \mathrm{~b}$ & $1.2 \mathrm{~b}$ & $2.3 \mathrm{~b}$ & $91.7 \mathrm{~b}$ \\
\hline
\end{tabular}

'Polaris was administered at $0700 \mathrm{~h}$, on day "0", as an aqueous foliar spray containing $3000 \mathrm{p} / \mathrm{m}$ active ingredient.

${ }^{2}$ Values in the same column (within experiments) bearing unlike letters differ significantly $(\mathrm{P}<.05)$.

attained under vastly reduced solar radiation (table 2 , experiment 2). While negative, these data are consistent with two kinds of prior evidence. First, a Polaris repression of the action spectra for net ${ }^{14} \mathrm{CO}_{2}$ assimilation (2), and second, a critical contribution by the period of leaf preillumination on the day that Polaris is applied. (3). From present evidence it appears that sugarcane photosynthesis rates in reduced sunlight are more than adequate to sustain chemical ripening without direct assistance from Polaris. That Polaris does not improve $\mathrm{CO}_{2}$ assimilation efficiency was also evidenced in experiment 3 , where light- 
deficient plants clearly needed "assistance", and where Polaris further repressed their already poor qualitative showing (table 2).

The inability of Polaris even to maintain control quality levels under heavy shade suggests that some aspect of its ripening action requires a critical minimum light intensity. This function may possibly involve the transport of assimilated carbon. Action spectra for ${ }^{14} \mathrm{C}$ translocation efficiency have shown an improved usage of visible radiation, in the blue-violet $(400 \mathrm{~nm})$ and green $(550-600 \mathrm{~nm})$ regions, by Polaris-treated cane (2). It is not known at this stage whether the shade material presently employed is preferentially selective against specific wavelengths.

TABLE 3.-Leaf canopy development for early-adult sugarcane treated with Polaris under variable sunlight regimes

\begin{tabular}{|c|c|c|c|c|c|c|}
\hline \multirow{2}{*}{$\begin{array}{l}\text { Exp. } \\
\text { no. }\end{array}$} & \multirow{2}{*}{ Light regime } & \multirow{2}{*}{ Treatment $^{2}$} & \multirow{2}{*}{$\begin{array}{l}\text { Leaves/ } \\
\text { plant }\end{array}$} & \multicolumn{3}{|c|}{ Leaf canopy values, at 21 days, for- } \\
\hline & & & & $\begin{array}{c}\text { Area/blade } \\
+3\end{array}$ & $\begin{array}{l}\text { Total blade } \\
\text { area }\end{array}$ & $\begin{array}{l}\text { Chloro- } \\
\text { phyll }\end{array}$ \\
\hline \multirow{5}{*}{2} & & & & $\mathrm{Cm}^{2}$ & $M^{2}$ & $M g / g$ \\
\hline & Full sun & Control & $6.2 \mathrm{~b}^{3}$ & 207 a & $1.28 \mathrm{a}$ & $1.58 \mathrm{~b}$ \\
\hline & & Polaris & $5.7 \mathrm{c}$ & $170 \mathrm{~b}$ & $0.97 \mathrm{~b}$ & $1.73 \mathrm{~b}$ \\
\hline & Intermediate shade & Control & $7.0 \mathrm{a}$ & $143 \mathrm{bc}$ & $1.00 \mathrm{~b}$ & $1.92 \mathrm{a}$ \\
\hline & & Polaris & $5.5 \mathrm{c}$ & $128 \mathrm{c}$ & $0.71 \mathrm{c}$ & $1.92 \mathrm{a}$ \\
\hline \multirow[t]{4}{*}{3} & Full sun & Control & $7.2 \mathrm{a}$ & 356 a & $2.57 \mathrm{a}$ & $2.42 \mathrm{a}$ \\
\hline & & Polaris & $4.5 \mathrm{~b}$ & $227 \mathrm{~b}$ & $1.02 \mathrm{~b}$ & $2.07 \mathrm{ab}$ \\
\hline & Heavy shade & Control & $4.0 \mathrm{~b}$ & $188 \mathrm{~b}$ & $0.75 \mathrm{bc}$ & $2.02 \mathrm{ab}$ \\
\hline & & Polaris & $2.4 \mathrm{c}$ & $177 \mathrm{~b}$ & $0.44 \mathrm{c}$ & $1.77 \mathrm{~b}$ \\
\hline
\end{tabular}

${ }^{1}$ Data were not obtained from experiment no. 1.

2 Polaris was administered at $0700 \mathrm{~h}$, on day " 0 ", as an aqueous foliar spray containing $3000 \mathrm{p} / \mathrm{m}$ active ingredient.

${ }^{3}$ Values in the same column (within experiments) bearing unlike letters differ significantly $(\mathrm{P}<.05)$.

An alternative consideration is the role that light may play in the preillumination of leaves about to receive Polaris. If, as previously suggested (3), a critical hormone-like substance is produced during this interval, full sunlight may provide excessive radiation while insufficient amounts are received under heavy shade. Experiments 2 and 3 were not ideally suited to handle this question. The shade-induced loss of green-leaf surface recorded at 21 days (table 3 ) was already underway after 12 days of shade equilibration. In this instance plants should be retained in a normal light regime until the day of treatment. It now appears that the low-intensity regimes attainable with controlled-environment chambers ( $3000-4000 \mathrm{fc}$ ) will be adequate to examine this type of light contribution. 


\section{RESUMEN}

Se evaluaron los efectos del Polaris sobre el crecimiento y la calidad del jugo de caña de azúcar adulta joven que recibió varios niveles de radiación solar. Se hicieron tres experimentos en los cuales las plantas, inicialmente propagadas en invernadero convencional, recibieron regímenes de sombra leve, intermedia y densa, con intensidades máximas de luz de 5,600, 2,300 y 450 pie-bujias, respectivamente. Se administraron tratamientos madurativos bajo luz solar completa (máximo diario $=9,600$ pie-bujías). El Polaris no requirió intensidades altas de luz solar para acción óptima ni mejoró la utilización de la planta bajo intensidades bajas de luz. La represión del crecimiento bajo sombra leve e intermedia fue comparable a la obtenida bajo luz solar completa. La sombra intermedia propició la calidad máxima del jugo, mientras que la sombra densa eliminó totalmente los efectos cualitativos de maduración. Los resultados sugieren que el Polaris requiere luz a intensidades relativamente bajas para los procesos de maduración, que no dependen directamente de la fotosintesis, y que puede ser usado efectivamente bajo una cubierta natural de nubes que reduce los niveles de radiación solar hasta 2,000 y 3,000 pie-bujias.

\section{LITERATURE CITED}

1. Alexander, A. G., and Montalvo-Zapata, R., Evaluation of chemical ripeners for sugarcane having constant nitrogen and water regimes. I. Growth, quality and enzymic responses of nine potential ripeners, Tropical Agr. 49: 35-44, 1973.

2. - and Biddulph, O., Effects of growth-regulatory chemicals on the action spectra for ${ }^{14} \mathrm{C}$ assimilation and transport in sugarcane leaves, J. Agr. Univ. P. R., 59(1): $15-25,1975$.

3. - Efficiency of chemical ripener action in sugarcane. I. Growth and quality responses to Polaris applied at different hours of the day, J. Agr. Univ. P. R., 60(4): 460-8, 1976.

4. - Efficiency of chemical ripener action is sugarcane. III. Growth and qualitative effects of Polaris as a function of the number and frequency of applications, J. Agr. Univ. P. R., 61(1): 11-18, 1977.

5. Arnon, D. I., Copper enzymes in isolated chloroplasts. Polyphenol oxidase in Beta vulgaris, Plant Physiol. 24: 1-15, 1949. 